\title{
Wavevector-Selective Nonlinear Plasmonic Metasurfaces
}

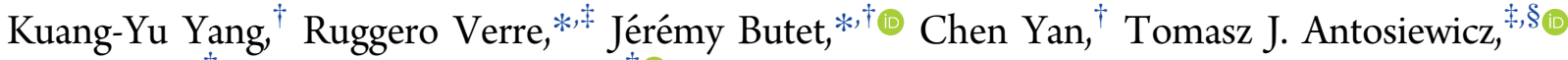 \\ Mikael Käll, ${ }^{\ddagger}$ and Olivier J. F. Martin ${ }^{\dagger}$ \\ ${ }^{\dagger}$ Nanophotonics and Metrology Laboratory, Swiss Federal Institute of Technology Lausanne (EPFL), 1015 Lausanne, Switzerland \\ ${ }^{\ddagger}$ Department of Physics, Chalmers University of Technology, 41296 Göteborg, Sweden \\ ${ }^{\S}$ Centre of New Technologies, University of Warsaw, Banacha 2c, 02-097 Warsaw, Poland
}

\section{Supporting Information}

\begin{abstract}
Electromagnetic metasurfaces with strong nonlinear responses and angular selectivity could offer many new avenues for designing ultrathin optics components. We investigated the optical second harmonic generation from plasmonic metasurfaces composed of aligned gold nanopillars with a pronounced out-of-plane tilt using a flexible nonlinear Fourier microscope. The experimental and computational results demonstrate that these samples function as wavevectorselective nonlinear metasurfaces, that is, the coherent second harmonic signal does not only depend on the polarization and wavelength of the excitation beam, but also of its direction of incidence, in spite of the subwavelength thickness of the active
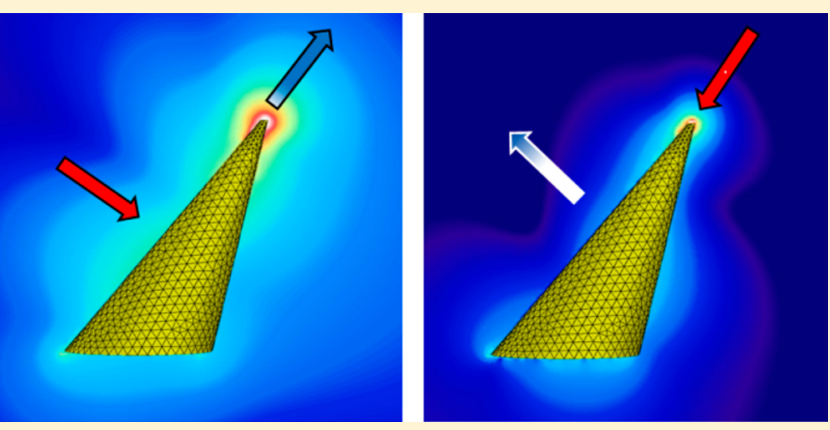
layer. Specifically, we observe that the nonlinear response can vary by almost two orders-of-magnitude when the incidence angle is changed from positive to negative values compared to the surface normal. Further, it is demonstrated that these metasurfaces act as a directional nonlinear mirrors, paving the way for new design of directional meta-mirrors in the nonlinear regime.
\end{abstract}

KEYWORDS: Metamaterials, nonlinear optical material, second harmonic generation, directionality, nonlinear plasmonics

\begin{abstract}
A rtificial electromagnetic metamaterials have led to the discovery of entirely new optical phenomena, ${ }^{1-4}$ such as negative refraction, 5,6 but the subwavelength scale $3 \mathrm{D}$ structuring required to make them has remained a challenge for applications, in particular in the visible spectral range. Twodimensional metamaterials, or metasurfaces, are much less demanding from a fabrication point-of-view, but they still offer an enormous potential for the development of new planar devices and functionalities. ${ }^{1-4}$ Metasurface research has led to the development of ultrathin planar lenses, ${ }^{7}$ Gaussian-to-Bessel beam transformers, ${ }^{8}$ broadband optical filters, ${ }^{9}$ quarter-wave plates, ${ }^{10}$ sensitive biosensors, ${ }^{11-13}$ and complex color routing devices, ${ }^{14}$ to name a few examples. However, the vast majority of metasurface research has considered the linear response regime, whereas investigations into nonlinear effects, such as optical frequency conversion, have remained scant. This is probably partly due to the limited current understanding of the mechanisms behind the nonlinear responses of the relevant meta-atoms (primarily dielectric and plasmonic nanoparticles), though important advances in this direction have been made recently. ${ }^{15-21}$ While optimizing the overall nonlinear optical conversion and controlling the nonlinear beam are important for the realization of practical applications, ${ }^{22-24}$ a subtle design of the coupling between the pump laser and a nonlinear metasurfaces is also essential for controlling the nonlinear response. However, due to their subwavelength dimensions, meta-atoms are more sensitive to the polarization state than to
\end{abstract}

the direction of propagation of the incident wave. For this reason, in-plane metamolecules have only been considered so far, meaning that the best coupling with the incident wave is in general reached at normal incidence and at the resonance wavelength. ${ }^{25-29}$ However, simultaneous control of the angular and spectral dependencies of nonlinear metasurfaces, extending the concept of wavevector filtering ${ }^{30}$ to the nonlinear regime, has not yet been reported, despite possible applications beyond those offered by diffractive optics. ${ }^{31}$

In this Letter, SHG from arrays of tilted gold nanopillars (see Figure 1) is investigated in detail. The SHG signal is measured using a nonlinear Fourier microscope, and the angular dependence of the second harmonic intensity is recorded for a wide range of illumination angles. It is found that nanopillars with various tilt angles allow different optical wavevector coupling, inducing a large and controllable asymmetry in the second harmonic $(\mathrm{SH})$ response. Finally, our metasurfaces are used as directional nonlinear mirrors extending the concept of reflective subdiffraction optics to the nonlinear regime. ${ }^{2}$

Several samples, homogeneous over a few $\mathrm{cm}^{2}$ with a density of $\sim 4$ nanocones $/ \mu \mathrm{m}^{2}$, were produced using a modified version of the hole mask colloidal lithography method (see Supporting Information for a detailed description). ${ }^{32}$ In brief, $100 \mathrm{~nm}$

Received: April 5, 2017

Revised: August 21, 2017

Published: August 22, 2017 
(a)

Negative angles Positive angles

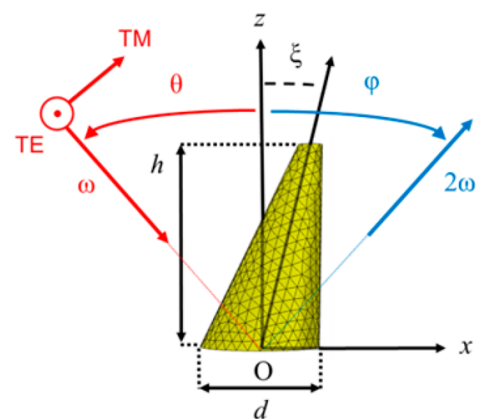

(b)

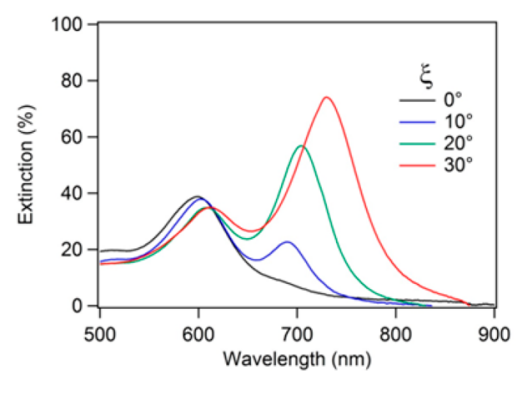

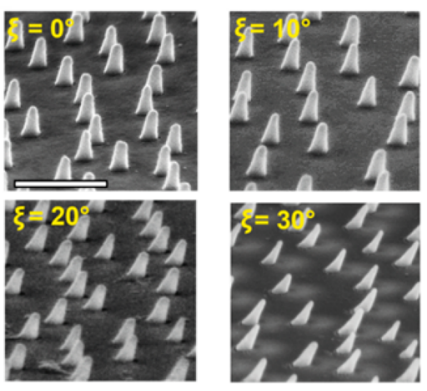

Figure 1. (a) Definition of the different parameters used in this Letter. The nanopillars are defined by their tilt angle $\xi$, their height $h$, and the diameter of their basis $d$. The angle $\theta$ corresponds to the illumination angle, and the angle $\varphi$ corresponds to the collection angle. (b) Extinction as a function of the incident wavelength for deposition angles ranging from $0^{\circ}$ to $30^{\circ}$. The height $h$ is $170 \mathrm{~nm}$, and the diameter $d$ is $100 \mathrm{~nm}$. The measurements are performed in air. (c) SEM images of the metasurfaces. The scale bar in the SEM image corresponds to 500 nm.

polystyrene beads were dispersed on top of a resist, and a 10 $\mathrm{nm}$ Au mask was evaporated. After the removal of the beads by tape stripping, the resist underneath the hole was etched by oxygen plasma to produce a large undercut, and $\mathrm{Ti}$ and $\mathrm{Au}$ layers were deposited at glancing angles followed by lift-off in acetone. During evaporation, the hole of the mask progressively shrinks, resulting in a tapering of the deposited structures. Scanning electron microscope (SEM) measurements confirm that the tilt angle $\xi$ is equal to the deposition angle. Figure 1c shows SEM images of the metasurfaces fabricated by the deposition of $170 \mathrm{~nm}$ of gold at different angles. The surface is covered by short-range ordered titled nanoparticles, conical in shape due to complete closing of the holed mask at the end of the evaporation process. The samples were first characterized by standard transmission spectroscopy in air at normal incidence in Figure 1b using linearly polarized light along the $x$-direction, as well as linear extinction and dipole moment calculations (Figures S1, S2, and S3). While the vertical nanocones exhibit a single resonance at $600 \mathrm{~nm}$, by progressively tilting the particles, an additional resonance appears at longer wavelengths. The behavior can be related to a lower symmetry of the system: in the case of the tilted nanocones, the superposition of in-plane and out-of-plane electron oscillations results in a tilt of the net dipole moment for these structures even at normal incidence illumination. In particular, from numerical simulations we extracted the net induced dipole moments $p$ along the $x$ - and $z$-directions and calculated the net tilt angle of the resulting dipole moments for normal illumination. For the nontilted structure $\left(\xi=0^{\circ}\right)$ the induced dipole is, evidently, parallel to the substrate. However, for $\xi \neq 0^{\circ}$, the dipolar orientation angle changes from close to horizontal to $60^{\circ}$ as the wavelength increases, this evolution being related to the deposition angle. Close to the high energy resonance, the dipolar angle is small, indicating an almost inplane resonance with small phase offset between the dipole moment in the $x$ - and $z$-directions. For the long wavelength resonance, the induced dipole is more vertical, and it does not align perfectly with the tilt of the nanostructure. The normal incidence illumination indicates that for tilted nanostructures the main axis of the system does not coincide with the experimental reference frame. As a consequence, the extinction for TM polarized light along the $x$-direction exhibits a strong asymmetry on the illumination angle, as shown in the Supporting Information (Figure S1).
Having characterized the linear responses of the fabricated arrays of nanopillars, we now turn our attention to their second harmonic responses. To do so, a nonlinear Fourier microscope has been built based on a femtosecond laser (Coherent Mira 900 with a pulse duration of $\sim 180 \mathrm{fs}$, a repetition rate of 80 $\mathrm{MHz}$, a central wavelength of $780 \mathrm{~nm}$ ), a dichroic mirror suitable for SHG as well as an oil immersion objective. This microscope is identical to a previous version developed in our lab for linear scattering measurements, which uses a white lamp and 50/50 beam splitter. This home-built nonlinear microscope permits recording the angular dependence of the second harmonic intensity in the $(O, x, z)$ plane as a function of the illumination angle; see Figure 2. Note that the incident wave propagates in the same plane. The experimental data obtained for a TM incident wave for the nontilted nanopillar are shown in the inset of Figure 2. The spectrum shows SHG only (no photoluminescence), and the observation of SHG is confirmed by the quadratic dependence of the recorded intensity on the pump power (Figure S4). The sample produces a second harmonic wave in the specular reflection direction, since the illumination angle and the second harmonic emission angle are always identical. ${ }^{33,34}$ In other words, the second harmonic wave corresponds to a very narrow light beam oscillating at the second harmonic frequency, and no diffuse SHG is observed. $^{33,34}$ This observation demonstrates the coherent nature of the second harmonic emission, in opposition with the hyper-Rayleigh scattering reported for nanostructured metal films. ${ }^{35,36}$ The divergence of the reflected second harmonic beam is estimated to be smaller than $3^{\circ}$; see Figure S5. This is a very important experimental observation, indicating that the studied arrays of gold nanopillars actually act as nonlinear metasurfaces and that the rules derived for SHG from surfaces and interfaces are also valid in this study. Actually, this behavior dramatically differs from common observations made for the SHG from isolated plasmonic nanostructures. ${ }^{37,38}$ In the case of single nanostructures, the second harmonic response is generally described using a multipolar expansion, ${ }^{17,39}$ providing a suitable framework for the description of the nonlinear scattering problem. If dispersion is negligible, that is, when the refractive index of the medium above the interface (immersion oil in the present case) is the same at the fundamental and second harmonic wavelengths, then the second harmonic emission angle is equal to the illumination angle. This means that each gold nanopillar is a source of a second harmonic wave and that the second harmonic signal observed in the far-field 
corresponds to the interference between all the emitted waves. When the illumination angle changes, the relative phase between the different second harmonic emissions evolves in a specific way, resulting in specular SHG. Since the same behavior has been observed for all samples measured, it is hence enough to study the $\mathrm{SH}$ specular reflection.

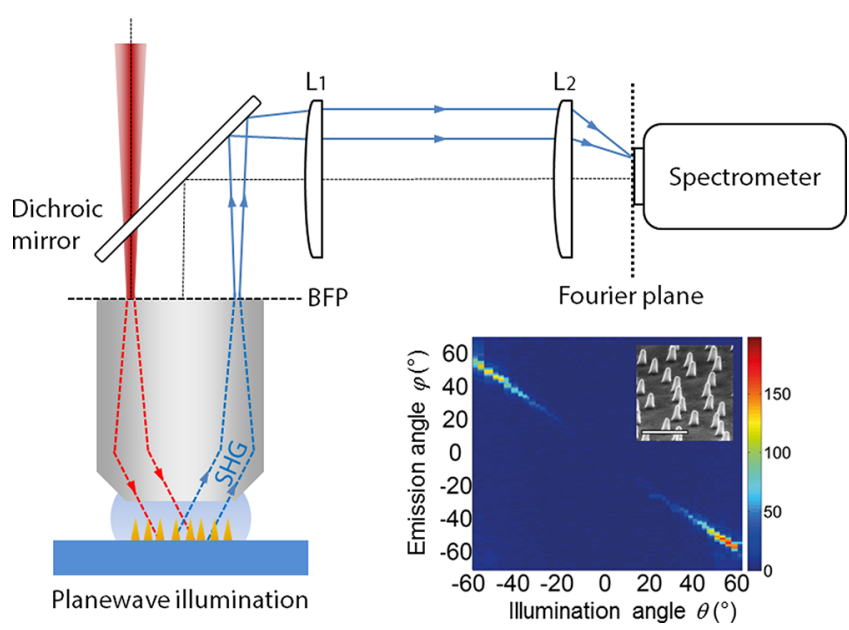

Figure 2. Diagram showing the illumination and collection experimental schemes. Inset: SHG as a function of the illumination angle for an incident wave in the $(O, x, z)$ plane for nontilted nanopillars. The nanopillars height is $170 \mathrm{~nm}$. The wavelength of the incident laser beam is $780 \mathrm{~nm}$. The scale bar in the SEM image corresponds to $500 \mathrm{~nm}$.

In Figure 3, both the linear response and the $\mathrm{SH}$ signal of the nanopillar metasurfaces with tilt angles $\xi$ ranging from $0^{\circ}$ to $30^{\circ}$ are investigated in a homogeneous environment $(n=1.5)$ for an incident wavelength $\lambda=780 \mathrm{~nm}$ and different illumination directions $\theta$. As anticipated from simple symmetry considerations, the second harmonic signal from nontilted nanopillars (with a vanishing tilt angle) is identical for negative and positive illumination angles. In this case, the maximal second harmonic intensity is obtained for the highest illumination angles reachable with the microscope objective $\left( \pm 60^{\circ}\right)$. The same effect has already been observed in SHG from gold nanotips ${ }^{40-42}$ and nanocones. ${ }^{43,44}$ However, as soon as the tilt angle increases, the second harmonic response is not the same for positive and negative illumination angles, and the second harmonic intensity is high when the incident electric field is parallel to the nanopillars axis. It is interesting to note that, contrary to the linear regime, ${ }^{45}$ a tilt angle as small as $10^{\circ}$ already results in a significant asymmetry in the $\mathrm{SH}$ response. In this case, there is a factor 3-difference between the maximal second harmonic intensity obtained for positive illumination angles and that for negative ones. To confirm that the wavevector dependency is solely due to the optical properties of the nanopillars, the SHG signal using TE illumination was also recorded, and a small symmetric signal was collected in this case (Figure 3).

To quantify the action of the nanopillar metasurfaces as nonlinear wavevector filters, an asymmetry parameter $\zeta$ is introduced as the ratio between the second harmonic intensities obtained for negative and positive illumination angles: $\zeta=$ $I_{\mathrm{SHG}}(-\theta) / I_{\mathrm{SHG}}(+\theta)$. Since measurements are performed in a reflection geometry, the asymmetry parameter hence permits us to assess the influence of the nanopillar tilt $\xi$ on the asymmetry of the nonlinear response both experimentally (as a function of the illumination angle $\theta$ ) and in simulations (as a function of the fundamental wavelength). As shown in the Supporting Information, Figure S7, the asymmetry parameter $\zeta$ reaches experimentally a value of about 80 , that is, a value more than 20 times larger than what is observed in transmission in linear optical measurements. This value can be further increased using an optimal pumping wavelength, by modifying the precise geometry of the system and by using objectives with larger numerical apertures.

To investigate the origin of the asymmetry in the second harmonic response, simulations of SHG from gold nanopillars with a tilt angle ranging from $0^{\circ}$ to $30^{\circ}$ have been performed using a surface integral equation method; ${ }^{46,47}$ see Figure 4. Single nanopillars embedded in an effective surrounding medium (effective refractive index $n=1.4$ ) are considered for all of the computations. For the computation details, the reader is referred to our previous publications describing the surface integral equation method for SHG from plasmonic nanostructures. ${ }^{47}$ Note that a surface contribution is considered, in line (a)
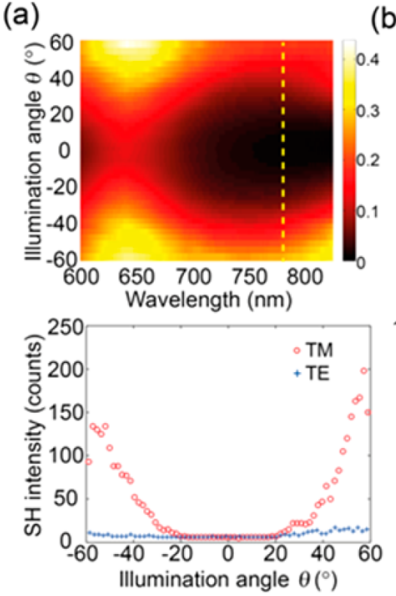

(b)
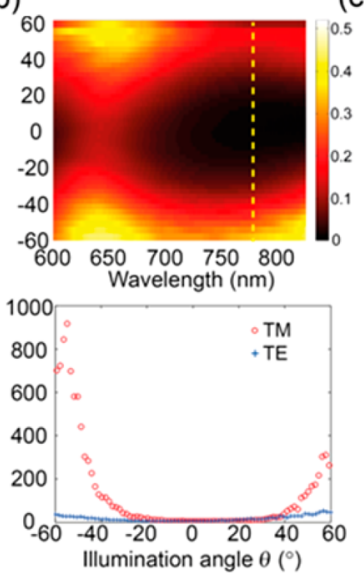

(c)
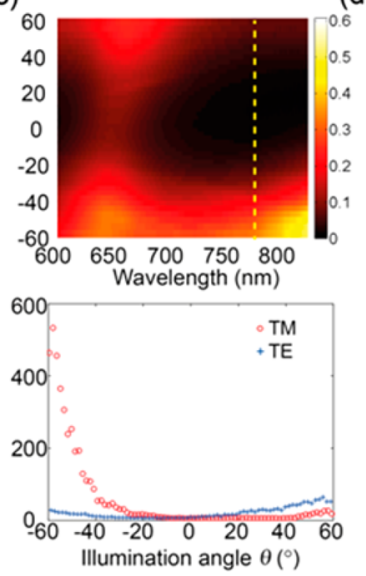

(d)

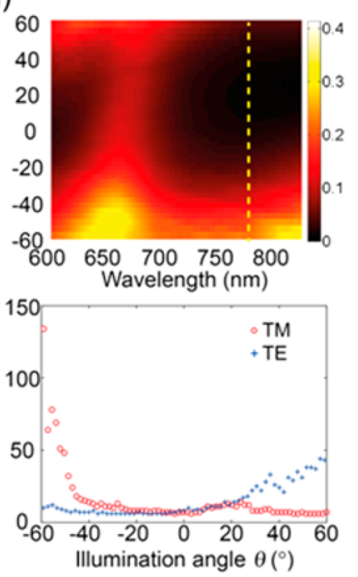

Figure 3. Reflection spectra (top panels) and angular dependences of the specular second harmonic intensity (bottom panels) for an incident wave in the $(O, x, z)$ plane with a TM polarization (red circles) and a TE polarization (blue crosses) (bottom panels). The nanopillars height is $170 \mathrm{~nm}$. The tilt angle is (a) $0^{\circ}$, (b) $10^{\circ}$, (c) $20^{\circ}$, and (d) $30^{\circ}$. For the measurement of the SHG, the wavelength of the incident laser beam is $780 \mathrm{~nm}$, indicated by the dashed yellow lines. 
(a)
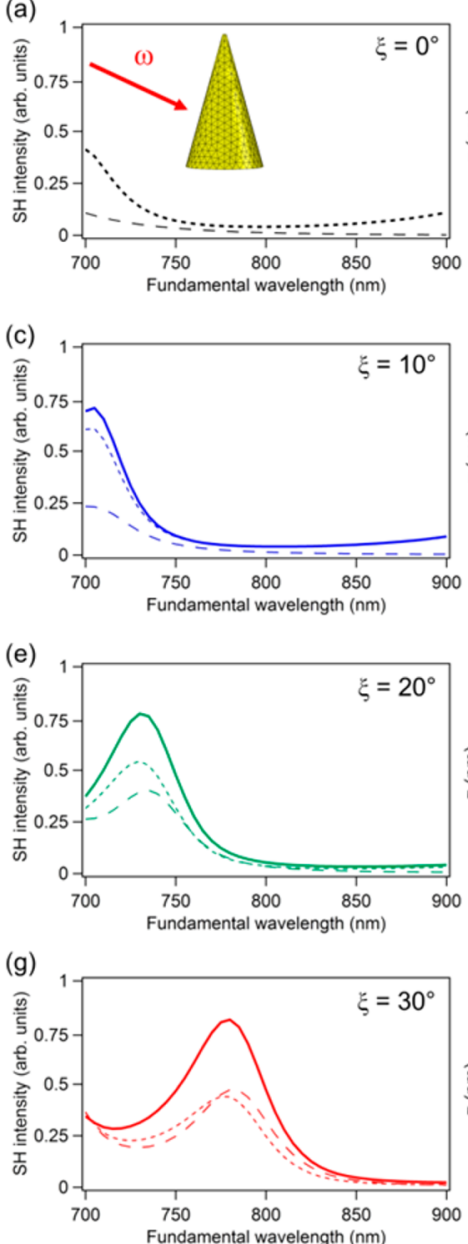
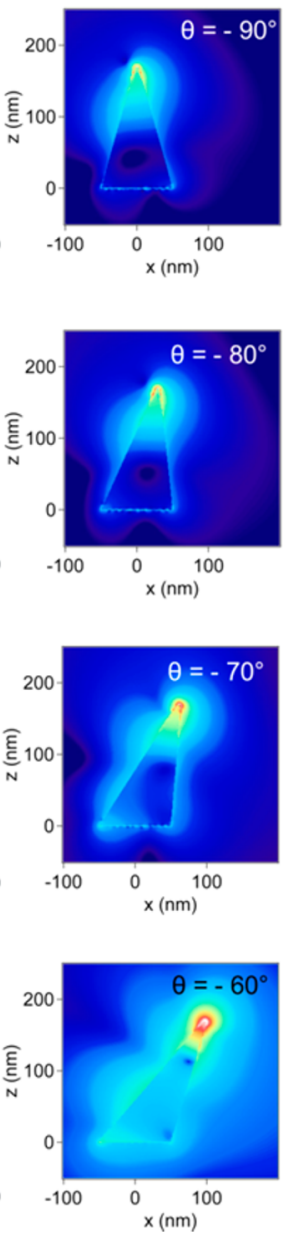

(b)
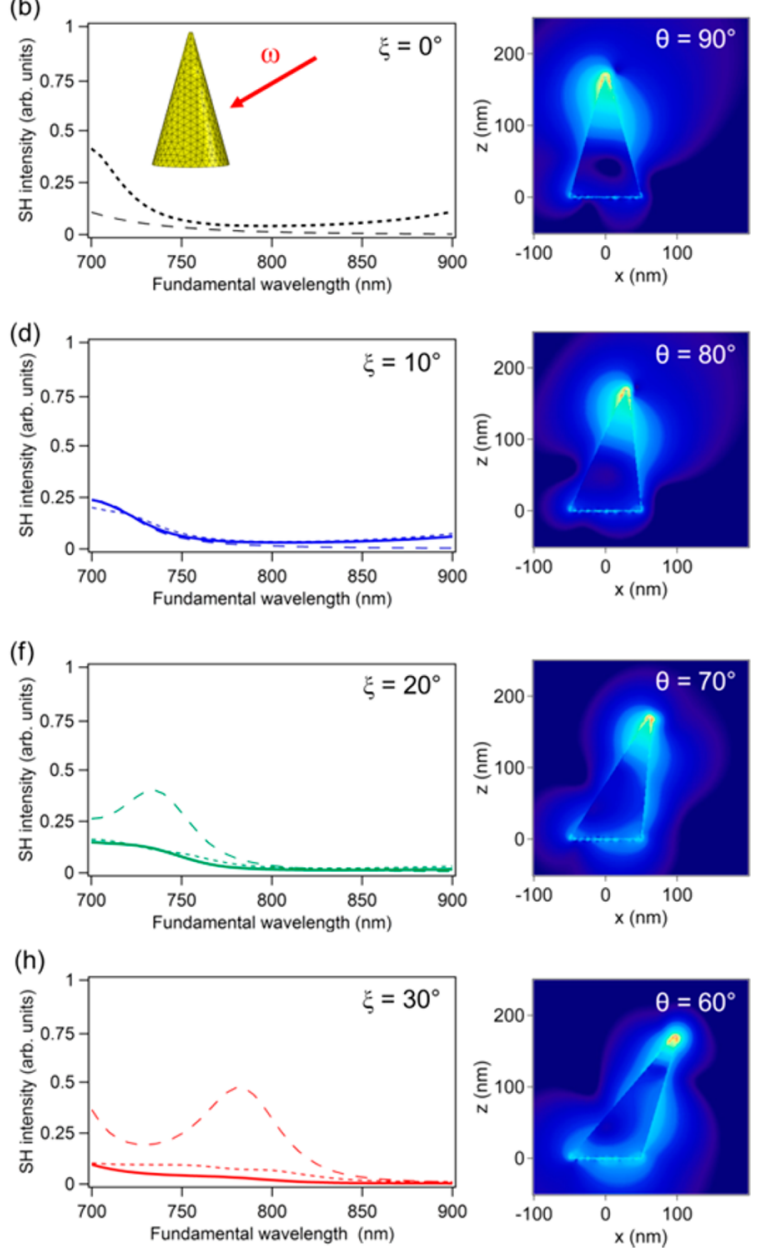

Figure 4. Calculated second harmonic intensity for single gold nanopillars with a tilt angle of (a, b) $0^{\circ},(\mathrm{c}, \mathrm{d}) 10^{\circ},(\mathrm{e}, \mathrm{f}) 20^{\circ}$, and (g, h) $30^{\circ}$. The results for negative and positive illumination angles are shown in the left and right columns, respectively. The dashed lines show the second harmonic intensity for a normal incident wave ( $z$-axis). The dotted lines show the second harmonic intensity for an incident wave propagating along the $x$-axis. The full lines show the second harmonic intensity for an incident wave polarized along the nanopillar axis (left column) or for illumination angles with the same norm and negative value (right column). The maps show the second harmonic intensity close to the nanopillars for the denoted illumination angle, corresponding to the full lines, and a fundamental wavelength of $780 \mathrm{~nm}$. The same colorscale is used for all the near-field distributions.

with recent experimental results. ${ }^{48,49}$ In the case of nanopillars with a vanishing tilt angle, the total second harmonic intensity is identical for incident waves propagating in the $+x$ - and $-x$ directions, as expected from symmetry consideration. However, the near-field distributions of the second harmonic intensity are not symmetric. This reveals that retardation plays an important role in the nonlinear response, despite the noncentrosymmetric shape of the studied nanostructures. As a consequence of retardation, second harmonic emission does not correspond to emission of a pure electric dipole but is multipolar in nature; see Figure S6. This point underlines that full-wave computations are essential for the full understanding of SHG from plasmonic nanostructures. ${ }^{50}$ In this framework, it is important to identify the position of the nonlinear sources over the nanopillar surface. The second harmonic intensity is the highest at the nanopillar apex, indicating that the strongest sources of SHG are localized in this area. The strong nonlinear polarization at the nanopillar apex is a direct consequence of the strong fundamental field enhancement, as discussed previously in refs 40-42. For all of the gold nanopillars, the second harmonic intensity is higher for an incident wave polarized along the nanopillar axis than for an incident wave polarized along the $x$ - or $z$-axis. The second harmonic intensity is maximal when the fundamental wavelength matches the resonant wavelength of the longitudinal mode. As the tilt angle increases, the effective length of the nanopillars increases, resulting in a redshift of the longitudinal mode. The computations reveal that the resonant wavelength is also an important parameter for maximizing the $\mathrm{SH}$ signal. Indeed, away from the resonant wavelength, the second harmonic intensity obtained for positive and negative angles is almost the same; see Figure 4. We fabricated nanopillars with different heights and verified that effectively the $\mathrm{SH}$ signal progressively increases once the resonance overlaps with the pump wavelength (see the Supporting Information, Figure S8).

So far, we have thus demonstrated that 3D nanopillars present an asymmetric $\mathrm{SH}$ emission depending on the tilt angle of the nanostructures. Now, we will make use of the large asymmetry parameter from the nanopillar arrays to realize a directional nonlinear mirror. To demonstrate the effect, a focused Gaussian beam with a diameter of few micrometers is used for illumination by enlarging the laser beam to fill the whole back aperture of the microscope objective; see Figure 5a. Indeed, such a laser beam can be decomposed into the sum of 
(a)
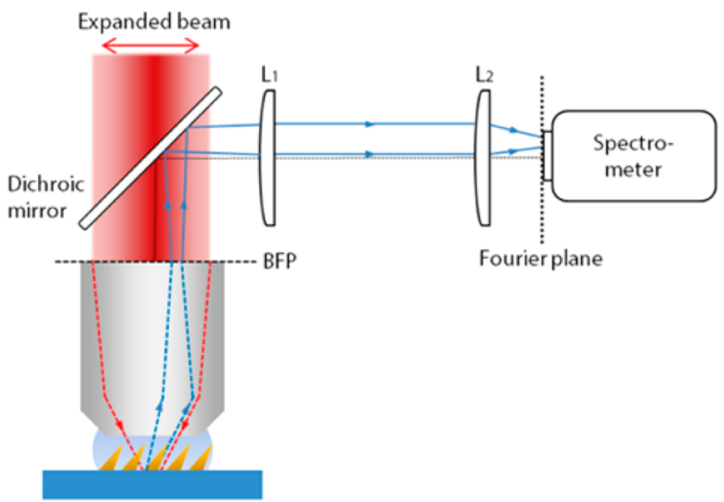

Focused beam illumination
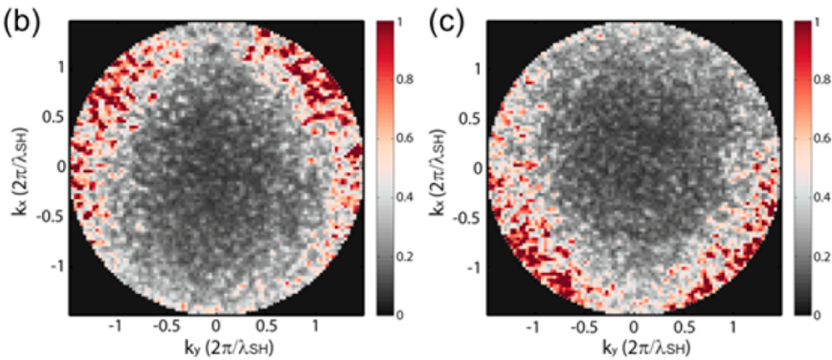

Figure 5. (a) Experimental configuration involving a focused beam illumination. Second harmonic far-field emission recorded in the Fourier plane for gold nanopillars with $d=100 \mathrm{~nm}$, height $h=130 \mathrm{~nm}$, and tilt angle $\xi=30^{\circ}$. The focused incident beam is obtained by enlarging the laser beam to fulfill the whole back aperture of the microscope objective. In panel $b$, the sample is oriented as described in Figure 1 , while in panel $c$, the nanopillars are pointing along the $-x$ direction.

incident planewaves with different wavevectors. ${ }^{51}$ The second harmonic emission from a standard $\mathrm{Ag}$ film has been first recorded. As a linearly polarized excitation was used, a symmetric two-lobe emission pattern was observed (Supporting Information, Figure S9). However, when the second harmonic far-field emission for gold nanopillars with the largest SH signal $\left(d=100 \mathrm{~nm}, h=130 \mathrm{~nm}\right.$, and tilt angle $\left.\xi=30^{\circ}\right)$ was recorded; a strong asymmetry in the nonlinear emission is clearly observed in Figure $5 \mathrm{~b}$, although a symmetric incident beam is used. To prove that this asymmetry is due to the sample properties, not to an experimental bias, the sample was flipped and measured again: in this case, the nonlinear emission pattern also flips (Figure 5c). The asymmetry of the nonlinear emission pattern for the metasurface is explained by wavevector selection occurring at the fundamental wavelength. Indeed, as demonstrated previously using a planewave excitation, only the incident conditions able to drive the surface plasmon resonances in the nanopillars give a significant second harmonic emission. In the present case, only a given part of the wavevectors composing the focused Gaussian beam indeed interacts with the sample, explaining the asymmetry in the second harmonic emission. It is worth noting that, due to the beam size reduction, fewer nanopillars are illuminated, and the nonlinear response is somehow between that of metasurface (specular reflection) and that of a single nanopillar (nonlinear scattering), explaining the observed two-lobe pattern in Figure 5.

In conclusion, SHG from arrays of tilted gold nanopillars has been investigated in detail using a flexible nonlinear Fourier microscope. The experimental results demonstrate that the

fabricated assemblies of gold nanopillars act indeed as wavevector-selective nonlinear plasmonic metasurfaces, beyond the nonlinear response expected for single and isolated nanostructures, but following the nonlinear reflection laws derived for nonlinear planar surfaces and interfaces. Nanocones with various tilt angles and heights have been considered, demonstrating how the illumination angle selectivity and the asymmetry in the second harmonic response can be controlled, emphasizing the importance of both the nanostructure shape and the plasmon enhancement. These observations paved the way for the realization of directional nonlinear plasmonic metamirrors.

\section{ASSOCIATED CONTENT}

\section{Supporting Information}

The Supporting Information is available free of charge on the ACS Publications website at DOI: 10.1021/acs.nanolett.7b01412.

Linear optical characterization in air, dipolar moment computations, computed second harmonic emission patterns from various nanocones, and linear and nonlinear measurements performed in the $(O, y, z)$ plane (PDF)

\section{AUTHOR INFORMATION}

\section{Corresponding Authors}

*E-mail: ruggero.verre@chalmers.se.

*E-mail: jeremy.butet@epfl.ch.

ORCID $\odot$

Jérémy Butet: 0000-0001-9598-9074

Tomasz J. Antosiewicz: 0000-0003-2535-4174

Olivier J. F. Martin: 0000-0002-9574-3119

\section{Author Contributions}

K.-Y.Y., R.V., and J.B. contributed equally to this work. K.-Y.Y. developed the nonlinear Fourier microscope, performed the measurement of the SHG from the metasurfaces, and edited the manuscript. R.V. fabricated the samples, performed both the linear optical characterization in air and the SEM images and edited the manuscript. J.B. supervised the development of the nonlinear Fourier microscope, performed the simulations of the SHG, and wrote the manuscript. C.Y. performed the linear optical characterization in oil and edited the manuscript. T.A. analyzed the linear response of the metasurfaces. M.K. and O.J.F.M. supervised the project and edited the manuscript.

\section{Notes}

The authors declare no competing financial interest.

\section{ACKNOWLEDGMENTS}

Funding from the Swiss National Science Foundation (grants 200020_153662 and 200021L_156184) and the European Research Council (ERC-2015-AddG-695206 Nanofactory) is gratefully acknowledged. This work was also supported by the Swedish Foundation for Strategic Research (SSF) and the Knut and Alice Wallenberg Foundation.

\section{REFERENCES}

(1) Kildishev, A. V.; Boltasseva, A.; Shalaev, V. M. Science 2013, 339, 1232009.

(2) Yu, N.; Capasso, F. Nat. Mater. 2014, 13, 139-150.

(3) Meinzer, N.; Barnes, W. L.; Hooper, I. R. Nat. Photonics 2014, 8, 889-898. 
(4) Jahani, S.; Jacob, Z. Nat. Nanotechnol. 2016, 11, 23-36.

(5) Pendry, J. B. Phys. Rev. Lett. 2000, 85, 3966-3969.

(6) Pendry, J. B.; Ramakrishna, S. A. J. Phys.: Condens. Matter 2003, $15,6345-6364$.

(7) Aieta, F.; Genevet, P.; Kats, M. A.; Yu, N.; Blanchard, R.; Gaburro, Z.; Capasso, F. Nano Lett. 2012, 12, 4932-4936.

(8) Pfeiffer, C.; Grbic, A. Phys. Rev. Lett. 2013, 110, 197401.

(9) Jiang, Z. H.; Yun, S.; Lin, L.; Bossard, J. A.; Werner, D. H.; Mayer, T. S. Sci. Rep. 2013, 3, 1571.

(10) Yu, N.; Aieta, F.; Genevet, P.; Kats, M. A.; Gaburro, Z.; Capasso, F. Nano Lett. 2012, 12, 6328-6333.

(11) Verre, R.; Maccaferri, N.; Fleischer, K.; Svedendahl, M.; Länk, N. O.; Dmitriev, A.; Vavassori, P.; Shvets, I. V.; Käll, M. Nanoscale 2016, 8, 10576-10581.

(12) Hakonen, A.; Svedendahl, M.; Ogier, R.; Yang, Z.-J.; Lodewijks, K.; Verre, R.; Shegai, T.; Andersson, P. O.; Käll, M. Nanoscale 2015, 7, 9405-9410.

(13) Svedendahl, M.; Verre, R.; Käll, M. Light: Sci. Appl. 2014, 3, e220.

(14) Yan, C.; Yang, K.-Y.; Martin, O. J. F. Light: Science \& Applications 2017, 10.1038/lsa.2017.17.

(15) Kauranen, M.; Zayats, A. V. Nat. Photonics 2012, 6, 737-748.

(16) Minovich, A. E.; Miroshnichenko, A. E.; Bykov, A. Y.; Murzina,

T. V.; Neshev, D. N.; Kivshar, Y. S. Laser Phot. Rev. 2015, 9, 195-213.

(17) Butet, J.; Brevet, P.-F.; Martin, O. J. F. ACS Nano 2015, 9, 10545-10562.

(18) Husu, H.; Siikanen, R.; Mäkitalo, J.; Lehtolahti, J.; Laukkanen, J.; Kuittinen, M.; Kauranen, M. Nano Lett. 2012, 12, 673-677.

(19) Czaplicki, R.; Husu, H.; Siikanen, R.; Mäkitalo, J.; Kauranen, M. Phys. Rev. Lett. 2013, 110, 093902.

(20) Klein, M. W.; Enkrich, C.; Wegener, M.; Linden, S. Science 2006, 313, 502-504.

(21) Kruk, S.; Weismann, M.; Bykov, A. Y.; Mamonov, E. A.; Kolmychek, I. A.; Murzina, T.; Panoiu, N. C.; Neshev, D. N.; Kivshar, Y. S. ACS Photonics 2015, 2, 1007-1012.

(22) Segal, N.; Keren-Zur, S.; Hendler, N.; Ellenbogen, T. Nat. Photonics 2015, 9, 180-184.

(23) Tymchenko, M.; Gomez-Diaz, J. S.; Lee, J.; Nookala, N.; Belkin, M. A.; Alù, A. Phys. Rev. B: Condens. Matter Mater. Phys. 2016, 94 214303.

(24) Wolf, O.; Campione, S.; Benz, A.; Ravikumar, A. P.; Liu, S.; Luk, T. S.; Kadlec, E. A.; Shaner, E. A.; Klem, J. F.; Sinclair, M. B.; Brener, I. Nat. Commun. 2015, 6, 7667.

(25) Lee, J.; Tymchenko, M.; Argyropoulos, C.; Chen, P.-Y.; Lu, F.; Demmerle, F.; Boehm, G.; Amann, M. C.; Alù, A.; Belkin, M. A. Nature 2014, 511, 65-69.

(26) Li, G.; Chen, S.; Pholchai, N.; Reineke, B.; Wong, P. W. H.; Pun, E. Y. B.; Cheah, K. W.; Zentgraf, T.; Zhang, S. Nat. Mater. 2015, 14, 607-612.

(27) Linnenbank, H.; Linden, S. Optica 2015, 2, 698-701.

(28) Keren-Zur, S.; Avayu, O.; Michaeli, L.; Ellenbogen, T. ACS Photonics 2016, 3, 117-123.

(29) Metzger, B.; Hentschel, M.; Giessen, H. ACS Photonics 2016, 3, $1336-1350$.

(30) Fedotov, V. A.; Wallauer, J.; Walther, M.; Perino, M.; Papasimakis, N.; Zheludev, N. I. Light: Sci. Appl. 2015, 4, e306.

(31) Czaplicki, R.; Kiviniemi, A.; Laukkanen, J.; Lehtolahti, J.; Kuittinen, M.; Kauranen, M. Opt. Lett. 2016, 41, 2684-2687.

(32) Verre, R.; Svedendahl, M.; Länk, N. O.; Yang, Z. J.; Zengin, G.; Antosiewicz, T. J.; Käll, M. Nano Lett. 2016, 16, 98-104.

(33) Bloembergen, N.; Pershan, P. S. Phys. Rev. 1962, 128, 606-622. (34) Bloembergen, N.; Chang, R. K.; Jha, S. S.; Lee, C. H. Phys. Rev. 1968, 174, 813-822.

(35) Bozhevolnyi, S. I.; Beermann, J.; Coello, V. Phys. Rev. Lett. 2003, 90, 197403.

(36) Stockman, M. I.; Bergman, D. J.; Anceau, C.; Brasselet, S.; Zyss, J. Phys. Rev. Lett. 2004, 92, 057402.

(37) Butet, J.; Duboisset, J.; Bachelier, G.; Russier-Antoine, I.; Benichou, E.; Jonin, C.; Brevet, P.-F. Nano Lett. 2010, 10, 1717-1721.
(38) Zhang, Y.; Grady, N. K.; Ayala-Orozco, C.; Halas, N. J. Nano Lett. 2011, 11, 5519-5523.

(39) Smirnova, D.; Kivshar, Y. S. Optica 2016, 3, 1241-1255.

(40) Bouhelier, A.; Beversluis, M.; Hartschuh, A.; Novotny, L. Phys. Rev. Lett. 2003, 90, 013903.

(41) Neacsu, C. C.; Reider, G. A.; Raschke, M. B. Phys. Rev. B: Condens. Matter Mater. Phys. 2005, 71, 201402.

(42) Anderson, A.; Deryckx, K. S.; Xu, X. G.; Steinmeyer, G.; Raschke, M. B. Nano Lett. 2010, 10, 2519-2524.

(43) Bautista, G.; Huttunen, M. J.; Mäkitalo, J.; Kontio, J. M.; Simonen, J.; Kauranen, M. Nano Lett. 2012, 12, 3207-3212.

(44) Reichenbach, P.; Horneber, A.; Gollmer, D. A.; Hille, A.; Mihaljevic, J.; Schäfer, C.; Kern, D. P.; Meixner, A. J.; Zhang, D.; Fleischer, M.; et al. Opt. Express 2014, 22, 15484-15501.

(45) Martin, O. J. F.; Girard, C. Appl. Phys. Lett. 1997, 70, 705-707.

(46) Mäkitalo, J.; Suuriniemi, S.; Kauranen, M. Opt. Express 2011, 19, 23386-23399.

(47) Butet, J.; Gallinet, B.; Thyagarajan, K.; Martin, O. J. F. J. Opt. Soc. Am. B 2013, 30, 2970-2979.

(48) Bachelier, G.; Butet, J.; Russier-Antoine, I.; Jonin, C.; Benichou, E.; Brevet, P.-F. Phys. Rev. B: Condens. Matter Mater. Phys. 2010, 82, 235403.

(49) Wang, F. X.; Rodriguez, F. J.; Albers, W. M.; Ahorinta, R.; Sipe, J. E.; Kauranen, M. Phys. Rev. B: Condens. Matter Mater. Phys. 2009, 80, 233402.

(50) Butet, J.; Martin, O. J. F. J. Opt. Soc. Am. B 2016, 33, A8-A15.

(51) Novotny, L.; Hecht, B. Principles of Nano-Optics; Cambridge University Press, 2006. 